\title{
Alguns esquemas entoacionais característicos da leitura de um texto por portugueses e brasileiros
}

\author{
António Romano \\ Università di Torino - Itália \\ Lurdes de Castro Moutinho \\ Universidade de Aveiro - Portugal
}

Résumé

Cette étude a pour but immédiat celui de mettre en évidence quelques éléments intonatifs particulièrement typés dans des productions linguistiques communes du Portugais parlé au Brésil (PB) par rapport aux schémas réalisés le plus communément - dans les mêmes conditions - par des locuteurs du Portugais européen (PE).

Le manque d'études dans ce domaine a été une des motivations de cette recherche.

Notre approche privilégie la recherche de configurations mélodiques associées à des figures expressives manifestées sur l'échelle des 
contours d'unité tonale et intonative avec un aperçu qui tienne en compte des variables de structuration plus globales.

Notre étude est basée sur un texte lu-interprété par 6 locuteurs (3 locutrices portugaises de Aveiro et 3 locuteurs brésiliens).

Nos résultats montrent qu'en PB émerge assez systématiquement profil final de modalité déclarative, présentant des caractéristiques qui peuvent donner lieu à une sorte de cliché mélodique. Ce contour, ancré à des rapports de hauteur très systématique, se manifeste par une tonie caractérisée par une brusque monté mélodique sur la préaccentuelle, suivie d'une diminution abrupte de hauteur qui se stabilise ensuite sur la voyelle accentuée, pour se réduire encore sur les voyelles postaccentuelles. Cette tonie est précédée souvent par une longue séquence quasi-monotone qui réalise une protonie plate assez aiguë, généralement inconnue au PE. 


\section{INTRODUÇÃO}

sta comunicação tem como principal objetivo evidenciar os elementos entoacionais, particularmente tipificados em produções linguísticas comuns, do português falado no Brasil (PB) e relacioná-los com enunciados produzidos por falantes do português europeu (PE). A escassez de estudos neste domínio foi a principal motivação para esta pesquisa.

Com efeito, para estas duas variedades do Português, para além da caracterização das particularidades no nível segmental, têm constituído objeto de estudo privilegiado, as diferenças evidentes ao nível da estrutura rítmica e da organização temporal (BARBOSA, 2000; FROTA \& VIGÁRIO, 2000 e 2001; GALVES et al, 2002). No entanto, e apesar de pesquisas já efetuadas neste domínio específico (MIRA MATEUS, 1990; MORAIS BARBOSA, 1994), uma comparação eminentemente entoacional entre o PE e o PB revela-se ainda incipiente.

Assim, e numa perspectiva de estudo da variação geolingüística, enquadrada num projeto mais vasto da análise prosódica das variedades românicas (ROMANO, 2001a, CONTINI et al. 2002), pretendemos testar macroíndices de diferenciação prosódica entre PE e PB.

Enquanto a maior parte dos estudos recentes sobre entoação se consagram, inexplicavelmente, à detecção de tipos acentuais, baseando-se unicamente em parâmetros de altura melódica (mesmo para as variedades em que os acentos quantitativos parecem também importantes), e em coerência com o nosso objetivo de estudar a entoação, a nossa perspectiva de análise privilegia a detecção e análise de configurações melódicas associadas a representações gráficas que se apresentam como significativas e que se manifestam através de uma escala de contornos de unidades tonal e entoacional, 
sem perder de vista outras variáveis que dão conta de uma estruturação mais global (cf. 't HART et al., 1990; ROMANO, 2001b).

A perspectiva de interpretação dos movimentos melódicos observados visa reconhecer uma estruturação dependente, não só de um certo número de restrições sintáticas, semânticas, pragmáticas e lógicas, mas também de parâmetros de estruturação e distribuição da informação, que podem fazer variar o contorno daí resultante, deixando, no entanto, a possibilidade de ter em conta contornos expressivos que o falante escolhe (ou não) manifestar.

Se, por um lado, esta perspectiva de análise segue as teorias mais confiáveis sobre a estruturação prosódica e sobre a relação entoação-sintaxe (MARTIN, 1977, ROSSI, et al. 1981, AUBERGÉ, 1991, HIRST \& DI CRISTO, 1998, ROSSI, 1997), por outro lado, não podemos, no entanto, negligenciar a existência de restrições puramente prosódicas, de restrições eufônicas e eurítmicas (cf. também MARTIN, 1997) e, evidentemente outros de natureza emocional (FONAGY, 1983, CAELEN-HAUMONT, 2002).

Com efeito, cada vez que uma mesma frase é produzida, entram em jogo todas as variáveis, com o objetivo de respeitar "os encontros estruturais”, tal como são definidos por Aubergé (AUBERGÉ, 1991), embora as mesmas variáveis sejam também manipuladas inconscientemente pelo falante que introduz informações extra ou paralingüísticas. Dentre elas, indícios mais ou menos evidentes e mais ou menos controláveis, da sua proveniência geo-sociocultural.

É nesta linha que se enquadra a nossa análise, cujo primeiro objetivo é testar o espaço de variabilidade de dois macro-subsistemas prosódicos do português (PE e PB) e de observar/descrever toda a variabilidade interna que estes agrupamentos podem apresentar.

Com esse fim, propomo-nos começar por recolher "melodias de fala" exploradas enquanto elementos de caracterização e identidade linguística do falante e, por isso, particularmente freqüentes nas produções dos indivíduos de diferentes comunidades. O objetivo é seguramente ambicioso, tanto mais que se inscreve no quadro de uma investigação tipológica que poderia exigir longas 
operações de reconhecimento prévio nas diferentes áreas lingüísticas, podendo os isófonos prosódicos não coincidirem com as tradicionais fronteiras dialetais.

No nosso caso, este pré-inquérito, apenas iniciado, permitiunos evidenciar somente um traço de diferenciação bastante estável. A identificação desse traço diferenciador é sempre possível - na ausência de um julgamento prévio, por parte de dialetófonos da área lingüística em questão -, se procedermos a um treino perceptivo de sensibilização para este tipo de fenômeno e na condição de encontrarmos uma amostragem de produções que contenha, com alguma clareza, esse tipo de traço.

O trabalho aqui apresentado tem por base um corpus reduzido, quase um estudo de caso, obtido a partir de um texto lido, de forma expressiva, por seis falantes: três portugueses e três brasileiros, devendo a sua análise permitir-nos encontrar algumas características de diferenciação entre o PE e o PB. Uma análise preliminar do referido corpus deixa já antever uma preferência por esquemas melódicos específicos, claramente distintos, apresentando-se mais ou menos estáveis, para as duas variedades, nas mesmas posições de produção e com estruturas segmental, sintática e logicopragmática similares.

\section{ENQUADRAMENTO TEÓRICO E METODOLOGIA}

Trabalhos específicos têm norteado a história de investigações prosódicas sobre o Português e que, ao serem perspectivados de forma distinta - descritiva, contrastiva ou de representação fonológica -, permitiram aprofundar diversos aspectos neste domínio, utilizando metodologias diversificadas (para o PE v. DELGADO MARTINS, 1982; para o PB v. RAMEH, 1962 e 1966 e, no âmbito de estudos mais gerais, mas marcados pela escolha clara de uma teoria, LOPEZ STRODT, 1979 et MAJOR COLEMAN, 1979). ${ }^{1}$

Testemunho de um interesse renovado neste domínio é a obra recente de D. Hisrt e A. Di Cristo (1998) que, utilizando as mesmas bases e a mesma metodologia de análise, apresentam um estudo para as 
diferentes línguas e seus espaços linguísticos respectivos e onde podemos encontrar duas seções especialmente consagradas à apresentação dos sistemas prosódicos do PE (da autoria de M. Cruz Ferreira) e do PB (da autoria de J.A. de Moraes). No entanto, e dado o objetivo da obra, poucas informações fonéticas foram previstas e, por isso, poucos elementos de comparação, neste domínio, estão disponíveis. Em segundo lugar, à parte o espaço utilizado para a apresentação de precisões terminológicas e uma escolha diferente relativamente aos aspectos aprofundados, também a diferente estrutura adotada pelos dois autores nessas seções consagradas ao português, dificultam a recuperação de informações essenciais quando o objetivo é o de uma análise entoacional comparada e numa perspectiva variacionista. Apesar disso, observações incontornáveis estão presentes na contribuição de De Moraes (cf. outras publicações do autor, cujas conclusões se encontram resumidas nesta referência), relativamente ao sistema prosódico do $\mathrm{PB}$, sempre sustentadas por dados quantitativos. ${ }^{2}$

Utilizando um método rigoroso que aplicou ao estudo e comparação dos sistemas fonéticos das diferentes línguas e variedades, o único investigador, tanto quanto nós sabemos, que tentou, até o momento, apresentar índices de diferenciação para o PE e o PB, parece ter sido o foneticista italiano L. Canepari (cf. CANEPARI, 1983 e 1985). Apoiando-se numa análise auditiva, a maior parte do tempo baseada sobre a sua própria percepção de um certo número de características, generalizadas a partir de um material diversificado, este autor soube resumir (no âmbito de publicações gerais), em alguns traços, as principais constantes que se podem esquematizar, quando de uma comparação ideal entre PE e PB. No seu percurso, seguindo uma tradição de análise entoacional de escola britânica, apresenta - sobre uma espécie de escala musical chamada tonograma - esquematizações de perfis melódicos tal como são percebidos pelo ouvido, com indicação de agrupamentos temporais, onde as proeminências são realizadas com uma certa estabilidade e, no caso das línguas com acento livre - acento lexical -, a indicação das posições dos segmentos acentuados. Geralmente, onde, tal como em 
outros modelos atualmente existentes, podemos distinguir posições nucleares e pré-nucleares, esta análise visa a descrição do desenvolvimento de uma fase preparatória, denominada protonia, encaminhando-se para uma fase terminal, tonia, marcada habitualmente por um perfil específico, como o apresentado nas figuras 1 e $2 .^{3}$

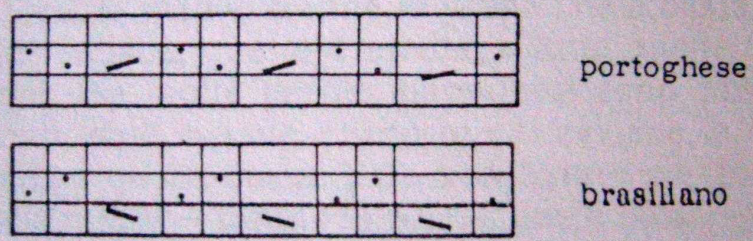

Fig. 1. Esquemas característicos de realizações da protonia em PE e PB, tal como foram descritos por L.Canepari (1985, p. 41).

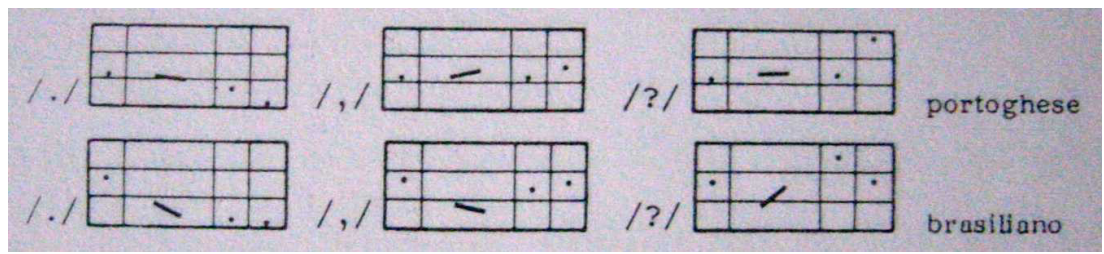

Fig. 2. Esquemas característicos de realizações de diferentes tonias em PE e PB, tal como foram descritos por L.Canepari (1985, p. 50).

Tendo em conta o que nós pudemos observar, esta divisão merece que se dê mais atenção ao desenvolvimento melódico na transição entre as duas partes - a tonia e a protonia - que constituem a intonia que revela, por si só, propriedades específicas. Um outro aspecto digno de maior atenção, negligenciado por uma escolha prévia de quantificação por níveis, diz respeito às relações melódicas a ser estudada em termos de configurações melódicas, ligadas a níveis freqüenciais. Estas podem determinar contornos musicais muito tipificados, mas que se tornarão imperceptíveis numa modelização por níveis ou por finais binários (cf. ‘t HART et al. 1990).

Por outro lado, um dos problemas que permanece é a generalização de fatos observados, com base em material variável, tendo-se, de início, efetuado uma escolha de fatores considerados responsáveis 
pela caracterização prosódica. Este tipo de abstração, pelo menos no nosso caso, conduz ao aparecimento de contornos estilizados, para os quais será necessário confrontar as duas dimensões de alinhamento e a amplitude.

Além disso, e ao invés do que acontece com as representações binaristas para a descrição de fenômenos prosódicos (focalizado/não focalizado, alto/baixo, etc.), quando as variáveis parecem, pelo contrário, ser do tipo linear ou pelo menos não-binárias (cf. CAELENHAUMONT, 2002, ROMANO, 2001b), tomamos outro procedimento. Decidimos não negligenciar o fato de que os contornos detectados e estudados, em função de uma diversa interpretação expressiva manifestada em diferentes momentos pelos falantes gravados, sejam o resultado de uma especificidade geoprosódica de um certo número de melodias específicas e de estratégias particulares de estruturação entoacional da informação (exemplos disponíveis para dialetos do PE, nos primeiros resultados do projecto AMPER-Portugal, v. MOUTINHO et al., 1999; para a comparação PE/PB, v. FROTA, 2002).

Apesar disso, a divisão protonia/tonia, que aplicamos a uma análise instrumental, revelou-se muito útil na comparação das diferentes realizações (inclusive o suporte constituído pelas análises já publicadas por Canepari), mas o material de que dispomos apenas nos permitiu confirmar uma parte desses esquemas.

As realizações por nós analisadas possibilitaram a observação de elementos importantes de caracterização que, dificilmente, poderiam manifestar-se em gravações laboratoriais, efetuadas com falantes profissionais, nos quais estes, certamente, reproduziriam a variedade de prestígio, controlando-a também no nível expressivo.

O nosso corpus reduzido, utilizado para este estudo comparativo, foi gravado a partir de um texto lido-interpretado por 6 falantes (3 portuguesas de Aveiro e 3 brasileiros, um do Rio de Janeiro, outro da Bahia e uma de São Paulo, atualmente residente em Aveiro). ${ }^{4}$ O texto gravado inspira-se num excerto de um curso de Português para aprendizes franceses (DIAS DA SILVA J. \& PARVAUX S., 1991), sendo constituído por nove unidades tonais. 
1. Meu amigo, os cruzados já acabaram.

2. Então você não sabe que até o nome da moeda varia no Brasil?

3. Antes de 1986 era o cruzeiro,

4. Depois o cruzado,

5. Em 1988 o cruzado novo...

6. E voltou a chamar-se cruzeiro em 1990.

7. Eles só trazem dólares americanos.

8. Nenhum brasileiro viaja com cruzeiros.

9. Você sabe qual é a cotação do dólar?

Para cada uma das realizações ( 1 a 3 para as 9 unidades indicadas e para cada um dos falantes dos dois grupos), seguimos as etapas de análise e de modelização utilizadas no âmbito do projeto AMPER, recorrendo especialmente a Speech Analyzer e Matlab. ${ }^{5}$ Assim, a análise previu:

- extração dos valores de $\mathrm{F}_{0}$;

- estudo da variação de $\mathrm{F}_{0}$ ao longo da produção de cada unidade tonal, subdividida em protonia e tonia;

- observação da influência do acento lexical, no interior de cada unidade tonal, por considerarmos ser um índice relevante no caso de línguas de acento móvel;

- detecção e modelização de melodias específicas das produções analisadas;

- comparação/interpretação dos resultados obtidos para cada uma das variedades em estudo.

Relativamente a este último ponto, que constitui o objetivo central desta pesquisa, ainda não podemos fornecer resultados, mesmo que parciais, a não ser os relativos a macroíndices que separam os dois supersistemas do PE e do PB. 


\section{INTERPRETAÇÃO DOS RESULTADOS}

Após uma observação atenta das curvas melódicas, as tendências intragrupo aparecem generalizadas, enquanto que as causas de diferenciação, entre as produções dos falantes do mesmo grupo, foram excluídas desta descrição. Através das realizações dos diferentes falantes, constatamos que é a primeira parte da unidade 1 (aqui indicada como 1aㅡ), com um esquema melódico do tipo vocativo ("meu amigo", facilmente transformável numa frase do tipo "oh meu amigo!"- vocativo, tomada de palavra, ironia, etc.) que apresenta uma maior variabilidade melódica. Os contornos das interrogativas (2. e 9.) revelaram-se particularmente decepcionantes - normalmente muito interessantes no plano de comparações geoprosódicas -, porque foram provavelmente interpretadas de forma bastante diferente, por cada um dos falantes. Mais estáveis, mas igualmente não muito marcadas no nível de uma diferenciação no plano regional dos enunciados, apresenta-se a evolução das curvas melódicas expressas em conformidade com as unidades 3., 4. e 5. Soluções bastante coerentes foram escolhidas, por parte dos falantes portugueses, para a realização dos esquemas declarativos (1b., 7., 8.), tendo-se revelado mesmo muito interessante a interpretação do enunciado 6.

Inscrita no quadro de uma progressão de mudança de movimentos, esta frase podia ser interpretada como uma forma excessiva, quase limite, no nível da sua produção: da mesma maneira, o falante do Rio, influenciado pela evidência referencial do conteúdo, carrega-a de uma certa ironia, realizando um esquema facilmente identificável. Este tipo de realização está também presente na fala da entrevistada de S.Paulo, embora de forma um pouco atenuada.

Este perfil de modalidade, que emerge com bastante sistematicidade em certas variedades do PB, manifesta-se por uma brusca subida melódica, sobre a pré-acentuada, seguida de uma diminuição abrupta de altura, estabilizando-se em seguida sobre a vogal acentuada, para se reduzir ainda nas vogais pós-acentuadas (encontramos aqui o contorno 
estilizado proposto por CANEPARI, 1983, v. fig. 2). Esta realização da tonia é precedida, freqüentemente, por uma longa seqüência quase monótona e que realiza um contorno final de protonia bastante agudo (v. fig. 3 e traçado em negrito na fig. 4). ${ }^{6}$ Este contorno, convertido num estilema prosódico típico do $\mathrm{PB},{ }^{7}$ sofreu diversas manipulações, para testarmos os aspectos que melhor o caracterizavam.

Após experiências informais de simulação por síntese de contornos diferentes, gerados a partir deste, uma certa rigidez específica foi observada: uma diminuição ou aumento de $\mathrm{F}_{0}$, superior a $5 \mathrm{~Hz}$, pode ser responsável pela perda da força deste contorno. Com efeito, observamos que, nas realizações menos expressivas, o desvio entre os elementos, caracterizados por uma certa relação com a altura média, diminui e que certos valores podem não ser atingidos, em diferentes momentos onde seriam esperados, tomando por base a realização mais expressiva de que dispomos.

A configuração mais caracterizadora do PB que encontramos apresenta-se, por isso, plana (correspondente à sequência monótona realizada, steady stretch), cerca de 4 qT ( 4 quartos de tom) acima da frequência média do falante, seguida de um pico (pré-acentual) a +8 qT (sempre relativamente à altura de referência), seguidamente assistimos a uma descida bastante abrupta de -8 qT, incidindo sobre a vogal acentuada (com maior duração), e uma descida gradual sobre as vogais pós-acentuadas, atingindo o valor máximo de -15 qT.

Numa simulação com uma melodia sintética, obtida a partir dos valores atingidos para o falante do Rio - o mais facilmente identificado - pareceu-nos que esta configuração passa por quatro níveis estáticos de referência, respectivamente a 180 Hz, 208 Hz, 128 Hz et 102 Hz, com uma oscilação entre os 3 a $4 \mathrm{~Hz}$. Evidentemente que, no que diz respeito ao contorno da frase espontânea, ele apresenta uma evolução dinâmica, com um alinhamento bem específico dos aspectos melódicos mais destacados com a estruturação segmental da frase.

De seguida, apresentamos, na fig. 3, o contorno melódico tal como foi medido para realizações em PB, com correspondências segmentais em transcrição fonética; na fig. 4, alguns contornos sobrepostos, 
extraídos de produções portuguesas e brasileiras, relativas a uma parte da protonia e da tonia da mesma frase e que mostram, também, uma certa intervariabilidade; finalmente, a fig. 5 apresenta uma comparação de versões estilizadas (cf. 't HART et al. 1990) de curvas melódicas realizadas com os mesmos esquemas entoacionais.

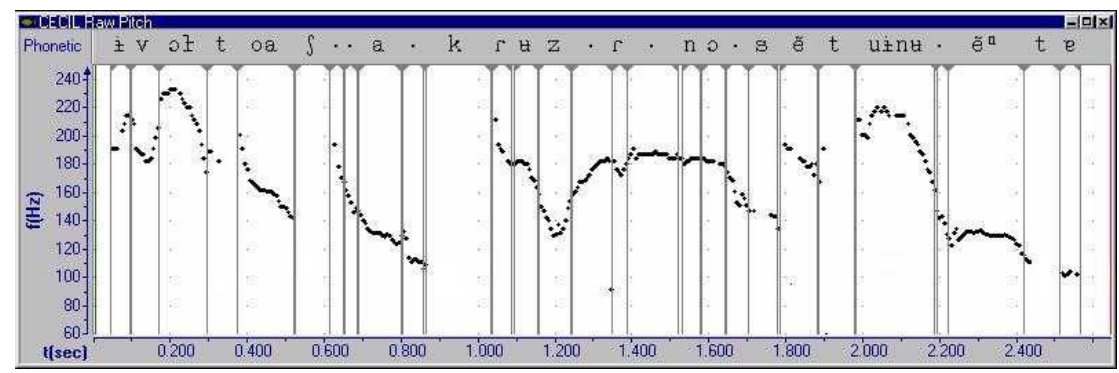

Fig. 3. Curva extraída para a unidade entoacional 6 ("E voltou a chamar-se cruzeiro em 1990") realizada enfaticamente pelo falante brasileiro do Rio.

[gráfico obtido com Speech Analyzer - SIL].
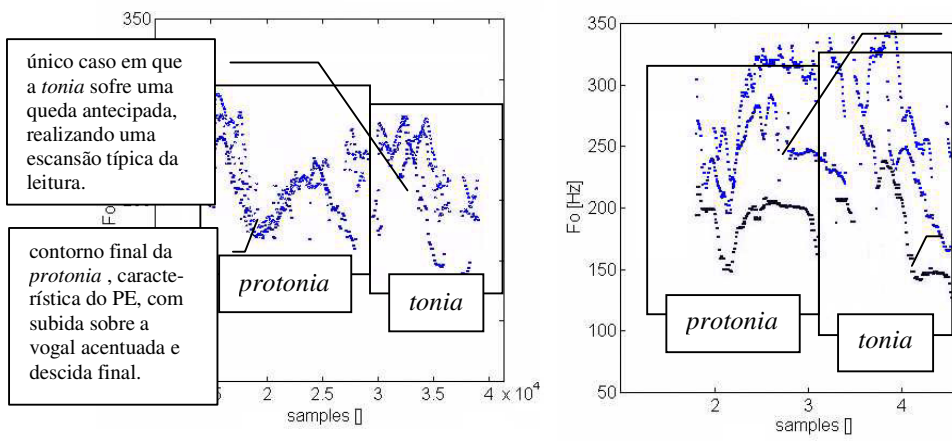

realização menos expressiva e menos conotada: a protonia não apresenta aqui o mesmo movimento muito caracterizador. esquema esteriotipado (tonia e final de protonia, cf. Fig 3): a vogal acentuada apresenta também uma configuração plana que se encontra ausente nos outros casos.

Fig. 4. Contornos sobrepostos de produções portuguesas

e brasileiras relativas à mesma passagem da Fig. 3 [gráfico obtido com Matlab - metodologia de extração de $\mathrm{F}_{0}$ do autor AR]. 

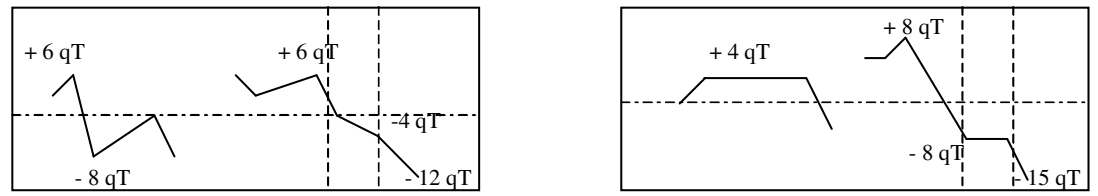

Fig. 5. Contornos estilizados de esquemas mais característicos de PE e PB em correspondência com (a.) a parte final da protonia e (b.) a tonia na interpretação dada por brasileiros e portugueses da mesma passagem da Fig. 3 [esquema feito manualmente]

\section{CONCLUSÕES}

Numa reflexão prévia sobre as razões que conduziam à diferença entoacional entre $\mathrm{PE}$ e $\mathrm{PB}$, constatamos, embora com um número reduzido de falantes, uma certa estabilidade na produção de alguns esquemas melódicos específicos aos dois supersistemas. A ordem da variabilidade por vezes constatada (quando se trata de pessoas do mesmo país, da mesma cidade e do mesmo nível social) apresenta como única explicação as diferenças nas escolhas de soluções expressivas para interpretar e transmitir o mesmo conteúdo. Esta estratégia utilizada por parte dos locutores deve ser, por isso, considerada prioritária nos estudos sobre a entoação; os investigadores que trabalham sobre este aspecto da estruturação linguística da mensagem, deveriam tê-la em consideração no momento da descrição da prosódia de uma qualquer variedade linguística. Certamente que outros fatores de variação dialetológica e sociolingüística deverão ser considerados (cf. MOUTINHO et al., 1999, ROMANO, 2001b), podendo estes funcionar, em alguns casos, como complemento de informação, no momento da leitura dos resultados.

Apesar de não termos perdido de vista essas variáveis, no decorrer desta pesquisa, o estudo contrastivo realizado permitiu-nos apenas destacar um esquema de variação entoacional, de entre o grande número que poderia ser identificado e organizado tipologicamente. Este esquema, do tipo declarativo, que aqui aparece com mais 
freqüência nas produções de um falante do Rio de Janeiro, nas marcadas por ênfase expressiva, poderia estar associado a um melisma identificativo, a uma espécie de melodia específica, uma entoação esteriotipada, de um PB, em oposição ao PE.

Concluímos, deixando aqui uma observação metodológica relativa às simplificações efetuadas sobre a representação das variáveis prosódicas. Na verdade, com os dados que analisamos, pareceu-nos inútil recorrer a um sistema de transcrição larga ou a um instrumento de etiquetagem prosódica do tipo interpretativo. Enquanto não tivermos ainda testado e inventariado as múltiplas possibilidades de variação, afigura-se-nos prematuro escolher um esquema arbitrário que generalizássemos como modelo de base. Descrever todos os pormenores, fazendo uma escolha meramente acidental, ou ainda mais discutível, introduzir técnicas de simbolização específicas, como se se tratasse de invariantes, conduzir-nos-ia, conseqüentemente, a interpretações errôneas dos resultados, daí a nossa opção.

Queremos também deixar claro que o estudo de caso, aqui apresentado, deve ser entendido como um estudo preliminar num domínio de pesquisa de grande complexidade. Aprofundar esta temática é um desafio que nos propomos assumir num futuro muito próximo.

\section{NOTAS}

${ }^{1}$ Para fenômenos específicos, analisados comparativamente entre o PE e o PB, trabalhos recentes abordam o problema de um ponto de vista instrumental e recorrendo a novos modelos (entre outros, FROTA \& VIGÁRIO, 2001; FROTA 2002).

${ }^{2}$ Num capítulo conclusivo, o autor acrescenta ainda considerações importantes sobre a variabilidade geográfica deste sistema, propondo, simultaneamente, um esboço de comparação entre certos traços de variedades geográficas do PB e do PE.

${ }^{3}$ Utilizaremos, ao longo de todo o texto, as expressões de Canepari - Tonia, Protonia -, na acepção que este autor lhes atribui, evitando a tradução por Tônica, etc., que não evocam o mesmo conceito. Na verdade, Tonia e Protonia referemsea agrupamentos silábicos, ao contrário do que acontece com os conceitos de Tônica e Pretônica que se referem apenas a vogais ou sílabas isoladas. 
${ }^{4}$ Outros falantes dos dois sexos (3 mulheres e 1 homem), de diferentes variedades do Português europeu (Lisboa, Barlavento, Açores), foram também gravados para validar a representatividade da leitura que foi feita do texto em que se baseia esta análise. Os dados destas produções constituem uma análise em curso, mas uma leitura sumária das curvas melódicas aqui apresentadas confirma as tendências observadas e resumidas neste artigo.

${ }^{5}$ Com a utilização de aplicações realizadas, no âmbito do referido projeto, por um dos autores deste artigo, AR.

${ }^{6}$ Este movimento bastante sistemático da protonia do PB escapa, no entanto, às descrições dadas por Canepari (1985); cf. Fig. 2.

${ }^{7}$ Há grandes possibilidades de reencontrar esta configuração melódica em entoações com conteúdo e conotação pragmático-lingüística similar no dialeto italiano de Gênova e nas variedades do italiano regional existente na Ligúria (Itália do Norte).

\section{REFERÊNCIAS BIBLIOGRÁFICAS}

AUBERGE, V. La synthèse de la parole: "des règles aux lexiques". 1991. Tèse (Doutoramento) - ICP-INPG/Université Stendhal et CRISS-Université Pierre Mendès-France, Grenoble. 1 v.

BARBOSA, P.A. Illuminating some Methodological Issues concerning Speech Timing Research from a Comparison between European and Brazilian Portuguese. Cadernos de Estudos Lingüísticos, n. 39, p. 41-50, 2000.

CAELEN-HAUMONT, G. Perlocutory values and functions of melisms in spontaneous dialogue. Proc. of the Int. Conf. Speech Prosody 2002, Aix-enProvence, p. 195-198, 2002.

CANEPARI, L. Phonetic notation / La notazione fonetica. Venezia: Cafoscarina, 1983. (vol. com dois cassetes).

CANEPARI, L. L'intonazione. Linguistica e paralinguistica. Napoli: Liguori, 1985.

CONTINI M.; LAI, J.P.; ROMANO, A.; ROULLET, S.; MOUTINHO, L. de C.; COIMBRA, R.L.; PEREIRA, B. U.; SECCA, Ruivo S. Un Projet d'Atlas Multimédia Prosodique de l'Espace Roman. Proc. of the Int. Conf. Speech Prosody 2002, Aixen-Provence, p. 227-230, 2002.

CRESTI E. Information and Intonational Patterning in Italian. In: FERGUSON, B. et al. (Ed.). Accent, Intonation et modèles phonologiques. Toronto: Mélodie, 1994. p. 99-140. 
CRUZ-FERREIRA, M. Intonation in European Portuguese. In: HIRST, D.; DI CRISTO, A. (Ed.). Intonation Systems. A Survey of Twenty Languages. Cambridge: Cambridge University Press, 1998. p. 167-178.

DE MORAES, J.A. Intonation in Brazilian Portuguese. In: HIRST, D.; DI CRISTO, A. (Ed.). Intonation Systems. A Survey of Twenty Languages. Cambridge: Cambridge University Press, 1998. p. 179-194.

DELGADO MARTINS, M.R. (1982). Sept Études sur la Perception. Accent et Intonation du Portugais. Lisboa: INIC, 1986.

DIAS da SILVA, J.; PARVAUX, S. Portugais. Pratiquer. Perfectionnement: pour maîtriser la langue et son environnement. Les langues pour tous, Presses Pocket, 1991. (vol. com três cassetes.)

FONAGY, I. La vive voix. Essais de Psychophonétique. Paris: Payot, 1983.

FONAGY, I.; FÓNAGY, J. L'intonation et l'organisation du discours. Bull. de la Société de Linguistique de Paris, LXXVIII, 1, p. 161-209, 1983.

FONAGY, I.; L.J., Boë; LUCCI, V. Incidence des variables situationnelles sur quelques paramètres de l'intonation. Actes des VIII'mes JEP-GALF. Aix-en-Provence, 1977, p. 13-19.

FROTA, S. The prosody of focus: a case-study with cross-linguistic implications. Proc. of the Int. Conf. Speech Prosody 2002. Aix-en-Provence, 2002. p. 319-322.

FROTA, S.; VIGÁRIO, M. Aspectos de prosódia comparada: ritmo e entoação no PE e no PB. Actas do XV encontro da APL. Braga, 2000. p. 533-555.

FROTA, S.; VIGÁRIO, M. On the correlates of rhythm distinctions: the European/ Brazilian Portuguese case. Probus, n. 13, p. 247-275, 2001.

GALVES, A.; GARCIA, J.; DUARTE, D.; GALVES, C. Sonority as a basis for rhythmic class discrimination. Proc. of the Int. Conf. Speech Prosody 2002. Aix-en-Provence, 2002, p. 323-326.

'T HART J.; COLLIER, R.; COHEN, A. A perceptual study of intonation. Cambridge, Cambridge University Press, 1990.

LOPEZ STRODT, B. The Sound Pattern of Brazilian Portuguese (Cariocan Dialect). 1979. Dissertation (Ph.D.) - University of California, Los Angeles.

MARTIN, Ph. Syntax and intonation: an integrated theory. Toronto Semiotic Circle, Monographs Working Papers and Prepublications, Victoria Univ., Toronto, 1977.

MARTIN, Ph. La Musique de la Phrase. In: GEZUNDHAJT, H.; MARTIN, Ph. (Ed.). Promenades en Phonétique, Toronto, Mélodie, p. 97-112, 1997. 
MAJOR COLEMAN, R. Prosody in Brazilian Portuguese phonology. 1979. Dissertation (Ph.D.) - The Ohio State University.

MIRA MATEUS, M. H. Fonética, Fonología e morfología do português. Lisboa: Universidade Aberta, 1990. (em col. com A. Andrade, M. Do Céu Viana \& A. Villalva).

MORAIS BARBOSA, J. Entoação e Prosódia. In: HOLTUS, G. et al. (Ed.). Lexikon der Romanistischen Linguistik, Band VI, n. 2, p. 243-248, 1994.

MOUTINHO, L. De C., COIMBRA, R.L., SECCA, R. S.; PEREIRA, B. U. (2001). Atlas Prosódico Multimédia: curvas de uma trajectória. Actas do XVI Encontro Nacional da Associação Portuguesa de Linguística. Coimbra, 1999, Colibri, 2001. p. 387-391.

RAMEH, Cl. A intonação no português do Brasil. Estudos Linguísticos, São Paulo, dez. 1966.

RAMEH, Cl. Contrastive analysis of English and Portuguese intonation. 1962. Thesis (Master) - Georgetown University.

ROMANO, A. Un projet d'Atlas Multimédia Prosodique de l'espace roman. Actes du XXIIİme CILFR. Salamanca, 2001a, en cours d'impression (éd. F. Sanchez Miret).

ROMANO, A. Applicabilité des systèmes de transcription et d'analyse de l'intonation aux cas de variabilité dialectale présentés par la situation géoprosodique italienne. Actes des Journées Prosodie 2001, Grenoble, 2001b, en cours d'impression (éd. A. Lacheret-Dujour).

ROSSI, M. Modèles de perception et de production de l'intonation. In: ROSSI, M. et al. (Ed.). L'intonation: de l'acoustique à la sémantique. Paris: Klincksieck, 1981.

ROSSI, M. L'intonation et l'organisation de l'énoncé. Pbonetica, n. 42, p. 140$144,1985$.

ROSSI, M. Is syntactic structure prosodically retrievable? Proc. of Eurospeech '97, Rhodes, 1997, p. 1-8. 